(2) Open Access Full Text Article

\title{
Posterior microphthalmos and papillomacular fold-associated cystic changes misdiagnosed as cystoid macular edema following cataract extraction
}

\author{
This article was published in the following Dove Press journal: \\ Clinical Ophthalmology \\ 7 January 2015 \\ Number of times this article has been viewed
}

\author{
Ahmad A Albar' \\ Sawsan R Nowilaty' \\ Nicola G Ghazi ${ }^{1,2}$ \\ 'Vitreoretinal Division, King \\ Khaled Eye Specialist Hospital, \\ Riyadh, Saudi Arabia; ${ }^{2}$ Department \\ of Ophthalmology, University \\ of Virginia, Charlottesville,VA, USA
}

Purpose: Novel spectral-domain optical coherence tomography (SD-OCT) findings in posterior microphthalmos (PM) include cystoid changes in the papillomacular fold (PMF). These changes may be misdiagnosed as cystoid macular edema (CME) and may trigger unnecessary treatment including intravitreal injections. We report a case that underscores this scenario aiming to increase awareness of this entity among ophthalmologists.

Method: A case report.

Results: A 25-year-old male presented with a history of clear lens extraction in both eyes as a refractive procedure. Postoperatively, he was diagnosed with CME and received five intravitreal bevacizumab injections in each eye as well as oral diamox for persistent cystic spaces noted on OCT. He was referred to our institution with the diagnosis of non-responding CME. A complete ophthalmic evaluation, including ultrasonography and SD-OCT, confirmed the diagnosis of PM and PMF with cystic cavities in the PMF. A fluorescein angiogram disclosed absence of macular leakage or optic nerve head staining indicating that the cystic cavities seen on SD-OCT are not due to postoperative CME but are rather the intrinsic cystic changes commonly seen in eyes with PM and a PMF. These cystic cavities were incorrectly thought to represent postoperative CME, which triggered the unnecessary treatment and lack of response.

Conclusion: Cystic-like cavities are a component of the PMF in PM eyes and may be misdiagnosed as CME, especially in a postoperative setting. This case underscores the importance of understanding the intrinsic features of the PMF in eyes with PM and may help increase awareness among ophthalmologists of such a potentially confusing scenario.

Keywords: macular edema, papillomacular fold, posterior microphthalmos

\section{Introduction}

Posterior microphthalmos (PM) is an autosomal recessive ocular condition ${ }^{1,2}$ characterized by high hyperopia, a normal appearing anterior segment of normal or subnormal dimensions, and a short axial length with posterior segment foreshortening. ${ }^{2-5}$ A retinal papillomacular fold (PMF) $)^{2-5}$ or wrinkles ${ }^{4-6}$ is a characteristic finding in most eyes with this condition. In about half of the cases, the PMF harbors cystoid changes. The latter can be either visible on fundus examination or detectable only on optical coherence tomography (OCT) imaging. ${ }^{6}$ These cystic changes may be misdiagnosed as cystoid macular edema (CME) and may trigger unnecessary treatment, including intravitreal injections. We report a case that underscores this clinical finding aiming to increase awareness of this entity among ophthalmologists.
Correspondence: Nicola G Ghazi King Khaled Eye Specialist Hospital, Al Urubah Road, Riyadh I |462,

Saudi Arabia

Tel +97l 526933400

Email nicola.ghazi@gmail.com 


\section{Case report}

A 25-year-old otherwise healthy male presented with a history of poor vision in both eyes for many years for which he had been wearing thick hyperopic glasses. Neither his non-consanguineous parents nor his five siblings had poor vision or obvious hyperopia. One year prior to his presentation at our institution, he underwent clear lens extraction with posterior chamber intraocular lens implantation in both eyes as a refractive procedure. His vision did not improve following surgery, and he was diagnosed with postoperative CME, namely, Irvine-Gass syndrome, as the cause of his poor visual outcome. During his postoperative course, the CME appearance persisted for which he received five intravitreal injections of bevacizumab and two transseptal triamcinolone injections for each eye. He was also treated with oral diamox with no improvement and was referred to us for non-responding CME.

On examination, the best-corrected visual acuity was $20 / 125$ in the right eye and 20/160 in the left eye. The intraocular pressure measured $12 \mathrm{mmHg}$ and $16 \mathrm{mmHg}$ in the right and left eyes, respectively. The anterior segment examination was unremarkable in both eyes except for well-centered posterior chamber intraocular lens bilaterally and mild posterior capsule opacification. The white-to-white horizontal corneal measurement was $11.5 \mathrm{~mm}$ in each eye. The posterior segment examination of both eyes showed mildly dilated and tortuous retinal blood vessels with a crowded optic disc and a horizontal PMF. PM was suspected, so an A-scan ultrasonography was performed, and it disclosed short globes with an axial length of $15.83 \mathrm{~mm}$ in the right eye and $15.87 \mathrm{~mm}$ in the left eye. Spectral-domain optical coherence tomography (SD-OCT) of the macula in both eyes showed a PMF (categorized as a high, inverted U pattern $)^{6}$ with cyst-like cavities in the inner nuclear and ganglion cell layers (Figure 1A and B). Fundus fluorescein angiography showed no optic nerve head staining or macular leakage (Figure 2). These findings confirmed the diagnosis of PM and bilateral PMF with intrinsic cysts that were incorrectly thought to represent postoperative CME (Irvine-Gass syndrome) and triggered unnecessary treatment. No further treatment for the PMF was recommended. A year later, the clinical and SD-OCT appearances of the PMF were unchanged (Figure $3 \mathrm{~A}$ and $\mathrm{B}$ ).

\section{Discussion}

Because the anterior segment in PM appears to be of normal or subnormal dimensions, PM may not be easily recognized unless an axial length measurement of the globe demonstrates the axial foreshortening, or the retinal PMF or wrinkles, characteristic of $\mathrm{PM},{ }^{2-6}$ is recognized during
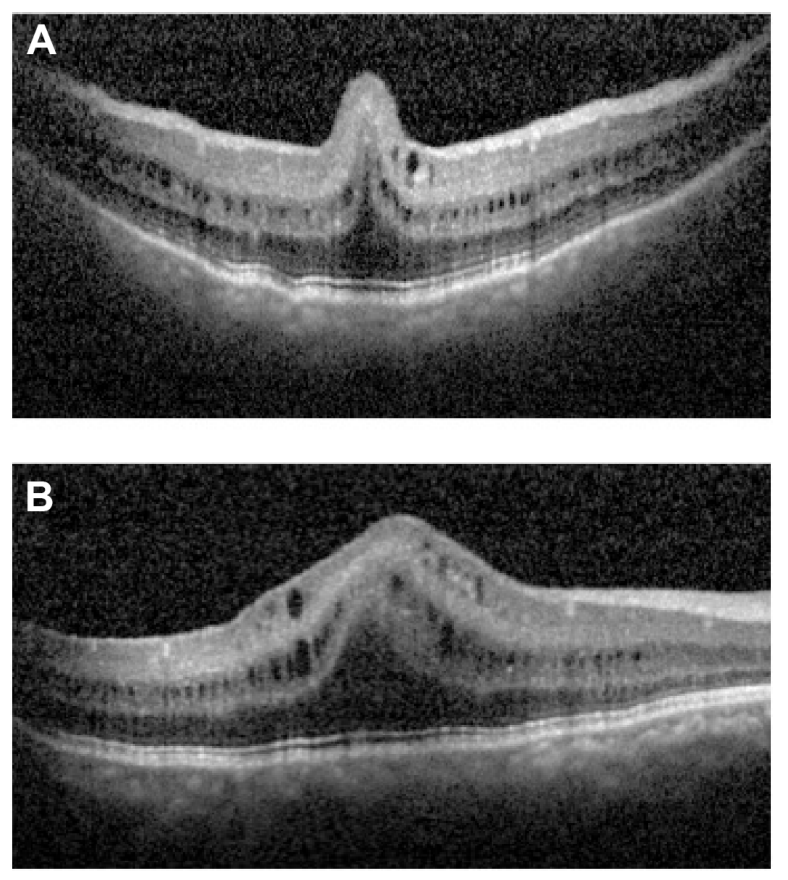

Figure I SD-OCT of the macula in the right eye at presentation. Notes: Both the vertical (A) and the horizontal (B) scan show a PMF with cystlike cavities in the inner nuclear and ganglion cell layers. The vertical scan (A) shows the high, inverted $U$ pattern of the PMF, and the horizontal scan (B) passing through the PMF clearly demonstrates the cystic cavities extending beyond the PMF. Note the preservation of the stratification of the retinal layers throughout the macula and the absence of involvement of the majority of the outer retina by the PMF. Abbreviations: SD-OCT, spectral-domain optical coherence tomography; PMF, papillomacular fold.

the fundus examination by ophthalmologists familiar with this condition. In a previous report, we described five types of folds, based on fundus examination and photography. ${ }^{6}$ In addition, based on SD-OCT vertical cuts passing through the PMF, the folds were categorized into one of the following five configurations: 1) an omega-shaped fold defined as a high, inverted U-shaped fold containing one or more large, centrally located, inner nuclear layer cyst(s);2) a high, inverted U-shaped fold; 3 ) an inverted V-shaped fold; 4) a low height, dome-shaped fold; or 5) only retinal surface wrinkles. ${ }^{6} \mathrm{We}$ also described some features intrinsic to the PMF, including sparing of the majority of the outer retinal layers, the absence of a foveal depression in most cases with preservation of the inner retinal layers within the PMF and beyond it, and cystic cavities of variable sizes in approximately $50 \%$ of the cases. ${ }^{6}$ These cavities were consistently located in the inner nuclear layer, but they were not always clinically visible. In some cases, the PMFs also harbored cystic cavities located in the ganglion cell layer. The latter were invariably visible on ophthalmoscopy. ${ }^{6}$ The case reported here harbored both a foveal elevation and visible cystic changes, clearly demonstrated in the ganglion layer 


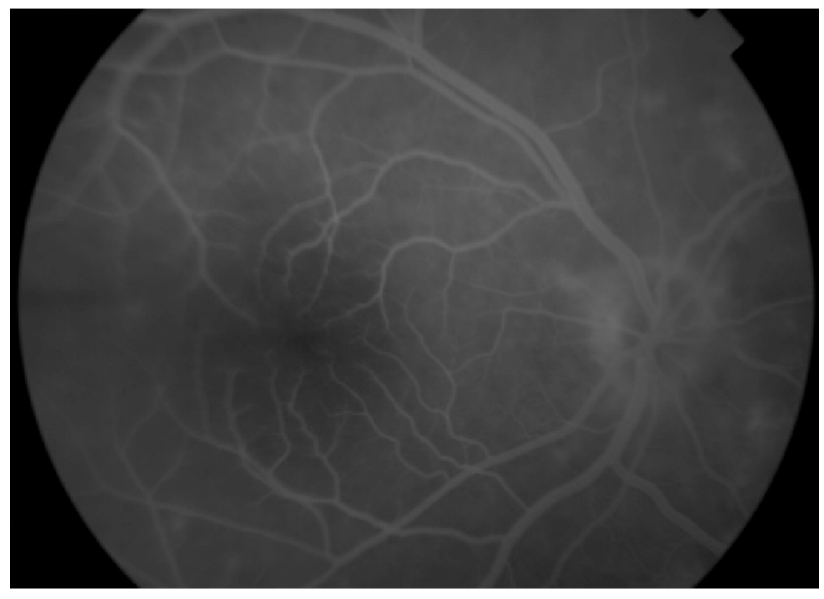

Figure 2 Fundus fluorescein angiography of the right eye in late phase shows no optic nerve head staining or leakage of dye in the macula.

Note: The haziness in the photograph is due to posterior capsular opacification.

on SD-OCT, which, together, were mistaken for CME by the treating ophthalmologist. The cystic cavities in this case represent, nevertheless, the characteristic intrinsic feature of the internal structure of the PMF in many eyes with PM. These cysts can be differentiated from postoperative CME in at least two ways. First, these are usually located in atypical layers of the retina for postoperative CME, namely, in the inner nuclear and ganglion cell layers, while the extracellular component of postoperative CME typically starts in the outer
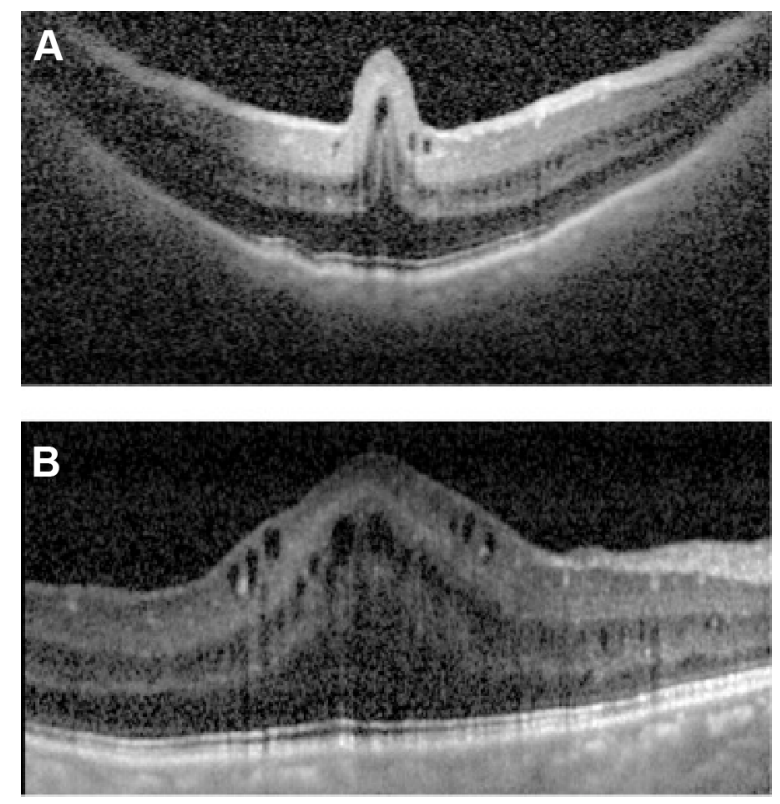

Figure 3 SD-OCT of the right eye I year after presentation.

Notes: The vertical (A) and horizontal (B) scans passing through the PMF show no change in the height, shape, or internal appearance of the PMF. The cystic cavities of inner nuclear and ganglion cell layers are unchanged.

Abbreviations: SD-OCT, spectral-domain optical coherence tomography; PMF, papillomacular fold. plexiform layer, and primarily affects the plexiform layers, before it may progress to involve the inner retinal layers. Second, these cystic-like changes are not associated with fluorescein angiographic leakage in the macular area or staining of the optic nerve head, features that characterize typical postoperative CME. The absence of fluorescein leakage in PMFs has been well described. ${ }^{3,6,8}$ In our case, the SD-OCT features were typical of PMF-associated cystic changes and the fluorescein angiogram was unremarkable with no macular leakage or disc staining, supporting the diagnosis of $\mathrm{PM}$ with PMF and excluding postoperative CME. In addition, the lack of any response to intensive and aggressive treatment as well as the stability of the cystic cavities on SD-OCT over almost a year of follow-up also supports a structural finding rather than postoperative CME.

In conclusion, this case report underscores the importance of understanding the salient features that may be associated with the PMF in eyes with PM in avoiding wrong diagnoses and unnecessary interventions as well as the potential risks that may be associated with them. Cystic changes seen on SD-OCT in eyes with PM after having undergone ocular surgery do not necessarily indicate postoperative CME. A careful evaluation, including fluorescein angiography, is critical in resolving the diagnosis.

\section{Disclosure}

In relation to this case report, the authors declare that there are no conflicts of interest.

\section{References}

1. Aldahmesh MA, Nowilaty SR, Alzahrani F, et al. Posterior microphthalmos as a genetically heterogeneous condition that can be allelic to nanophthalmos [letter]. Arch Ophthalmol. 2011;129:805-807.

2. Nowilaty SR, Khan AO, Aldahmesh MA, Tabbara KF, Al-Amri A, Alkuraya FS. Biometric and molecular characterization of clinically diagnosed posterior microphthalmos. Am J Ophthalmol. 2013;155: 361-372.

3. Boynton JR, Purnell EW. Bilateral microphthalmos without microcornea associated with unusual papillomacular retinal folds and high hyperopia. Am J Ophthalmol. 1975;79:820-826.

4. Spitznas M, Gerke E, Bateman JB. Hereditary posterior microphthalmos with papillomacular fold and high hyperopia. Arch Ophthalmol. 1983;101:413-417.

5. Khairallah M, Messaoud R, Zaouali S, Ben Yahia S, Ladjimi A, Jenzri S. Posterior segment changes associated with posterior microphthalmos. Ophthalmology. 2002;109:569-574.

6. Nowilaty SR, Mousa A, Ghazi NG. The posterior pole and papillomacular fold in posterior microphthalmos: novel spectral-domain optical coherence tomography findings. Ophthalmology. 2013;120(8): 1656-1664.

7. Fine BS, Brucker AJ. Macular edema and cystoid macular edema. Am J Ophthalmol. 1981;92:466-481.

8. Kim JW, Boes DA, Kinyoun JL. Optical coherence tomography of bilateral posterior microphthalmos with papillomacular fold and novel features of retinoschisis and dialysis. Am J Ophthalmol. 2004;138:480-481. 


\section{Publish your work in this journal}

Clinical Ophthalmology is an international, peer-reviewed journal covering all subspecialties within ophthalmology. Key topics include: Optometry; Visual science; Pharmacology and drug therapy in eye diseases; Basic Sciences; Primary and Secondary eye care; Patient Safety and Quality of Care Improvements. This journal is indexed on

PubMed Central and CAS, and is the official journal of The Society of Clinical Ophthalmology (SCO). The manuscript management system is completely online and includes a very quick and fair peer-review system, which is all easy to use. Visit http://www.dovepress.com/ testimonials.php to read real quotes from published authors. 